Nonlinear Processes in Geophysics, 12, 725-732, 2005

SRef-ID: $1607-7946 / \mathrm{npg} / 2005-12-725$

European Geosciences Union

(C) 2005 Author(s). This work is licensed

under a Creative Commons License.

\title{
Dissipation scales in the Earth's plasma sheet estimated from Cluster measurements
}

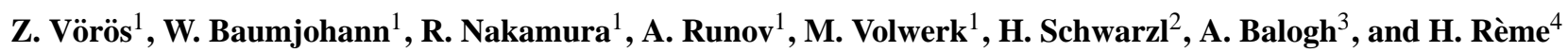 \\ ${ }^{1}$ Space Research Institute, Austrian Academy of Sciences, Graz, Austria \\ ${ }^{2}$ University of California, Los Angeles, USA \\ ${ }^{3}$ Imperial College, London, UK \\ ${ }^{4}$ CESR/CNRS, Toulouse, France
}

Received: 18 April 2005 - Revised: 8 July 2005 - Accepted: 8 July 2005 - Published: 27 July 2005

Part of Special Issue "Nonlinear and multiscale phenomena in space plasmas"

\begin{abstract}
In order to estimate the dissipation time-scale in magnetic turbulence in the plasma sheet a novel method is introduced for classification of velocity dependent patterns of two-point probability density functions' shapes near their maxima. For the first time, we provide evidence for Reynolds number (velocity) dependent widening of the inertial range in the plasma sheet. Since spectral widening of the inertial range is a generic feature of many turbulent flows, its examination can facilitate the recognition of intermittent turbulence in the plasma sheet.
\end{abstract}

\section{Introduction}

High-speed plasma flows called bursty bulk flows (BBFs) play a key role in the flux and energy transport in the magnetotail (Baumjohann et al., 1990; Angelopoulos et al., 1992; Schödel et al., 2001). Among other things, BBFs are the potential sources of multi-scale intermittent turbulence in the plasma sheet (Borovsky et al., 1997; Angelopoulos et al., 1999). Unlike the corroborated importance of turbulence in the solar wind (e.g. Tu and Marsch, 1995; Bruno et al., 2004; Horbury and Balogh, 2001), however, charcteristics and physical consequences of the bulk flow associated plasma sheet turbulence are still under debate (Borovsky et al., 1997; Greco et al., 2000; Neagu et al., 2002; Borovsky and Funsten, 2003; Volwerk et al., 2003, 2004; Klimas et al., 2004; Vörös et al., 2003, 2004b; Weygand et al., 2005). Non-steady and transitory features of BBFs and the associated motion of boundaries make difficult to accomplish a thorough statistical analysis of fluctuations (Vörös et al., 2004a). Therefore the linkage between the observed features of plasma sheet turbulence and the current understanding of turbulent flows

Correspondence to: Z. Vörös

(zoltan.voeroes@oeaw.ac.at) and their generic physical consequences is weak. In fact, the majority of rapid flows stays uninteruptedly at high-speed levels for only a few seconds. Neither the typically observed groups of high-speed intermittent flows last longer than some minutes (Baumjohann et al., 1990). Since plasma velocity measurements are typically available with time resolution of $\sim 4 \mathrm{~s}$ any relevant velocity-based statistical approach is hamstringed from the very beginning.

Phenomenological models of fluid turbulence, however, are based on velocity statistics. The turbulent energy flux in wavenumber space is also expressed in terms of velocity moments (Frisch, 1995). Following Kolmogorov phenomenology, velocity fluctuations in fluids are associated to different wavenumber ranges or scales ranging from the largest, energy-containing bulk flow scales to the smallest scales where substantial part of the flow energy is dissipated. For large enough Reynolds numbers the energy containing scales and the dissipation scales are clearly separated with a self-similar inertial range in between, where dissipation is supposed to be negligible and energy cascades towards large wavenumbers governed by nonlinear interactions. The width of the inertial subrange increases as the Reynolds number increases, which means that the energy containing large scales are separated further from the small scales (Saddoughi and Veeravalli, 1994; Frisch, 1995). The main goal of this paper is to check the Reynolds number dependence of properly defined small scales in the plasma sheet turbulence. The widening of the inertial subrange and the occurrence of multi-scale couplings may provide substantial evidence that the observed fluctuations are associated with turbulence rather than with waves. In the latter case spectral transfer of energy is absent and each timescale depends only on a few nearby wavenumbers (e.g. Oughton, 2003).

MHD turbulence in the plasma sheet is more complex than the outlined cascade picture would suggest. For example 
dissipation can be significant even within the inertial range of scales (Borovsky and Funsten, 2003) or direct interaction between large and small scales is possible without developing into a full cascade (Chang, 1999; Consolini and Chang, 2001). One might still expect changes in the character of cross-scale coupling, a shift of the dissipation scale or a properly defined small-scale, when the Reynolds number or the magnetic Reynolds number increases.

Different types of Reynolds numbers can be derived for the flows in the plasma sheet (Borovsky and Funsten, 2003) corresponding to different aspects of turbulent flows. In this paper we will not estimate the actual value of the Reynolds number in the plasma sheet, but, making a number of simplifications, only its relative changes will be considered. The flow Reynolds number we will consider is a dimensionless number $R=V L / v$, where $V$ is the average flow velocity, $L$ is the typical large spatial scale of the flow and $v$ is the kinematic viscosity. We suppose that, in the plasma sheet within the distances $X_{G S M} \in(-14,-20) R_{E}$ the viscous forces are not changing substantially. The width of the flow channel, which represents the typical large-scale of the flow in the expression of the Reynolds number, was estimated on the basis of statistical analysis of Cluster spacecraft multipoint measurements by Nakamura et al. (2004). It was shown that the full width of the flow channel is $2-3 R_{E}$ in the "dawndusk" direction and 1.5-2 $R_{E}$ in the north-south direction. The largest variations occur in the bulk flow velocity which can change from tens of $\mathrm{km} / \mathrm{s}$ to more than $1000 \mathrm{~km} / \mathrm{s}$ in the plasma sheet. Therefore, we consider $L / \nu \sim$ const. and suppose that for increasing bulk velocities a widening of the inertial subrange can be expected. Consequently, instead of analysing the Reynolds number dependence of turbulent small scales, we can investigate their bulk velocity dependence. In fact, high velocity flow associated widening of the inertial range towards small scales in magnetic turbulence was observed by Vörös et al. (2004b) and Volwerk et al. (2004). Nevertheless, the actual bulk velocity dependence of small-scale turbulence was not investigated.

In this paper we present detailed analysis of the distinct multi-scale properties of two-point probability density function (PDF). We will explore the small-scale behaviour of magnetic fluctuations in response to the changes in largescale bulk flows. The reason for using PDFs rather than spectral or wavelet methods for studying the small scales is simple. Spectral methods are affected by multiplicative bias (Vörös et al., 2004b) while wavelet methods are much more time consuming than the time-domain estimation of PDFs.

\section{Data sets and methods}

In order to study the bulk velocity (Reynolds number) dependence of small-scale magnetic fluctuations in plasma sheet turbulence we analyse $67 \mathrm{~Hz}$ resolution magnetic data from the Cluster fluxgate magnetometer (FGM) (Balogh et al., $2001)$ and the spin-resolution $(\sim 4 \mathrm{~s})$ velocity data from the Cluster Ion Spectrometry (CIS/CODIF) experiment (Rème et al., 2001). The selected intervals are from 2001, when the Cluster spacecraft were within the GSM coordinates $X_{G S M} \in(-14,-20) R_{E}$ and $Y_{G S M} \in(-10,10) R_{E}$. Moreover, we require $\left|B_{X}\right| \leq 20 \mathrm{nT}$ to ensure all data are taken from or close to the plasma sheet.

Burst mode magnetic field data from the Cluster spacecraft allow a wide statistical analysis and an immersion into scales or frequency ranges which are not available in velocity measurements during the short intervals of intermittent flows. As for the problem of missing small-scale velocity statistics, we note that a complete characterization of MHD turbulence requires not only the statistical description of the velocity field but also a statistical study of other measurable quantities, e.g. magnetic field, density or temperature fluctuations. So the analysis of magnetic field statistics is reasonable by its own. Nevertheless, magnetic field fluctuations can be used as a proxy for velocity statistics. Actually, it was shown by Bershadskii and Sreenivasan (2004) that the statistical scaling properites of the magnitude of the magnetic field in MHD flows resemble, in the inertial range, the scaling properties of passive scalars.

In this paper we will use a non-parametric method for the estimation of PDFs from magnetic data. The widely used histogram method estimates PDFs directly from time series $\left(X\left(t_{i}\right), i=1, \ldots, n\right)$, splitting the range of the data into equalsized bins of width $2 h$ ( $\pm h$ vicinity of a bin's center). Then the number of data points falling into each bin is counted and divided by the total number of observations $n$. In a more compact form the estimator of the density can be written as (Silverman, 1986)

$$
P(X)=\frac{1}{n h} \sum_{i=1}^{n} w\left(\frac{X-X\left(t_{i}\right)}{h}\right)
$$

where the weight function (rectangular window) $w$ is defined through

$w(s)= \begin{cases}0.5, & \text { if }|s| \leq 1, \\ 0, & \text { otherwise }\end{cases}$

It implies summing up "boxes" of width $2 h$ and height $(2 n h)^{-1}$, instead of counting the observations $X\left(t_{i}\right)$. The rectangular weight function, however, yields discontinous estimations. Therefore in this paper we will use the so-called kernel method (Silverman, 1986), which provides smoother estimations of the PDFs than the histogram method. The only difference is that now, $w=K$, and $K$ is not a rectangular window, but a a smooth symmetric PDF, in this paper the normal density. It satisfies the condition $\int_{-\infty}^{\infty} K(X) d X=1$. Instead of non-smooth "boxes", smooth "bumps" centered at the observations are summed up. The kernel function $K$ determines the shape of the bumps, while $h$ determines their widths.

Our goal is to analyse the scale evolution of PDFs. Therefore, it is essential to check the robustness of estimations against $h$ and $n$. In the following, we introduce multi-scale PDFs in the plasma sheet and perform the necessary tests for robustness of PDF estimations using a long enough interval of magnetic data. Then, for properly chosen $h$ and $n$, we analyse the scale-dependence of the shape of PDFs. 


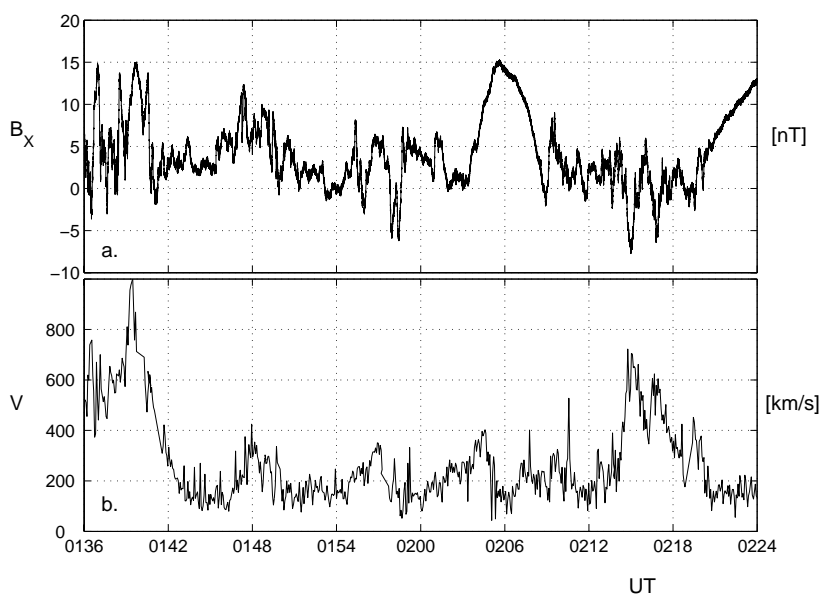

Fig. 1. Time series of (a) $B_{X}$ component of the magnetic field, (b) plasma velocity, measured by the Cluster spacecraft s/c 3 on August 2001, 01:36-02:24 UT.

\section{Scale-dependent probability density functions}

Unlike power spectra, multi-point PDFs can reveal statistical intermittency of fluctuations in turbulence. This manifests itself in the increased probability of large non-gaussian deviations and letokurtic shape of distributions. Intermittency and the departure from Gaussianity is stronger at small spatial or temporal scales, while largely separated uncorrelated points exhibit two-point PDFs close to the normal distribution. In order to demonstrate the evolution of PDFs over scales we chose the interval from 01:36 to 02:24 UT on 27 August, 2001. The magnetic $\left(B_{X}\right)$ and velocity $(V)$ data from s/c 3 are depicted in Figs. 1a and 1b. Figure 2 shows the multiscale behaviour of the amplitude of magnetic fluctuations. The "time scale" $\tau$ is introduced through differentiation

$\delta B=B(t+\tau)-B(t)$

where $B$ is the magnitude of the magnetic field and PDFs $(P(\delta B, \tau)$, Eq. 1) corresponding to the time delays $\tau=0.03-1000 \mathrm{~s}$ are shown in Fig. 2. In this way, as usual in space physics applications (e.g. Sorriso-Valvo et al., 1999), time series obtained from a single spacecraft are used for mapping the spatial structure of turbulence. Whenever the spatial fluctuations on a scale $l$ pass over the spacecraft faster than they typically evolve in time, this approach can be used. One can find a more profound discussion of the problem of spatial versus time scales in Borovsky et al. (1997); Borovsky and Funsten (2003); Vörös et al. (2004b). Since instead of spatial structures time-shifted structures will be considered throughout the paper, the inertial range or the dissipation range of scales will denote the corresponding time scales hereinafter. Specifically, the inertial range of scales will correspond to the range of scales over which self-similar multiscale fluctuations in frequency or wavelet-scale domain are present (not shown). The dissipation time-scale, $\tau_{d}$, will refer to a $\tau$, where self-similarity is broken and multi-scale fluctuations stop.

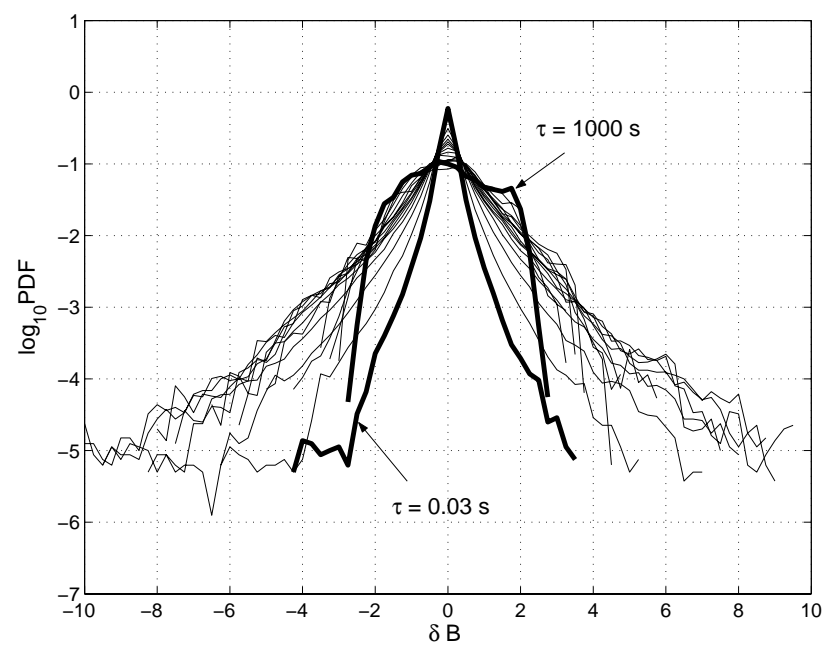

Fig. 2. Scale-dependent two-point PDFs. Small time-scale PDFs are more leptokurtic (peaked), large time-scale PDFs are close to the Gaussian distribution.

In terms of statistical physics, small-scale intermittency emerges as a consequence of the underlying non-extensivity and nonlinearity in space plasma turbulence (Leubner and Vörös, 2005a,b), therefore the corresponding statistical moments, e.g. the peakedness or kurtosis of PDFs are also dependent on the Reynolds number or bulk velocity (e.g. Porta et al., 2001). When the time series are too short, however, finite size effects might lead to divergence of high-order moments (e.g. Vörös et al., 2004a), circumventing any reliable estimation of large deviation statistics which characterizes the tails of PDFs. In principle, for processes exhibiting selfsimilarity, any point on the PDF curves can be used for the analysis of the scaling features of fluctuations. Since the central part of a distribution is statistically the most accurate part, one can analyse the scale dependence of PDFs, $P(\delta B, \tau)_{\max }$. Because of the finite accuracy of magnetic field measurements the peaks of distributions $\left(P(\delta B, \tau)_{\max }\right)$ may not be an optimal choice. Therefore points near $P(\delta B, \tau)_{\max }$ are recommended for use in statistical analysis (Hnat et al., 2002). A powerful test for self-similarity consists of rescaling the multi-scale PDFs on the basis of a power-law

$P(\delta B, \tau) \sim \tau^{-\alpha}$

determined directly from the raw PDFs (e.g. Sornette, 2000). Here $\alpha$ is the scaling index which can be used for rescaling the raw PDFs. In the solar wind, the power-law in Eq. (3) and prevailing self-similarity can be robustly observed e.g. in case of the magnetic energy density or the Poynting flux, whilst the fluctuations of the bulk speed and the magnitude of magnetic field exhibit multifractality (Hnat et al., 2002, 2003). We will show that the short (6 min) slow-flow associated intervals in the plasma sheet exhibit a power-law scaling within the scale-range of magnetometer noise. Therefore, the above-mentioned rescaling technique cannot be used for straightforward analysis of self-similar turbulence in our case. But still, one can find particular velocity-dependent 


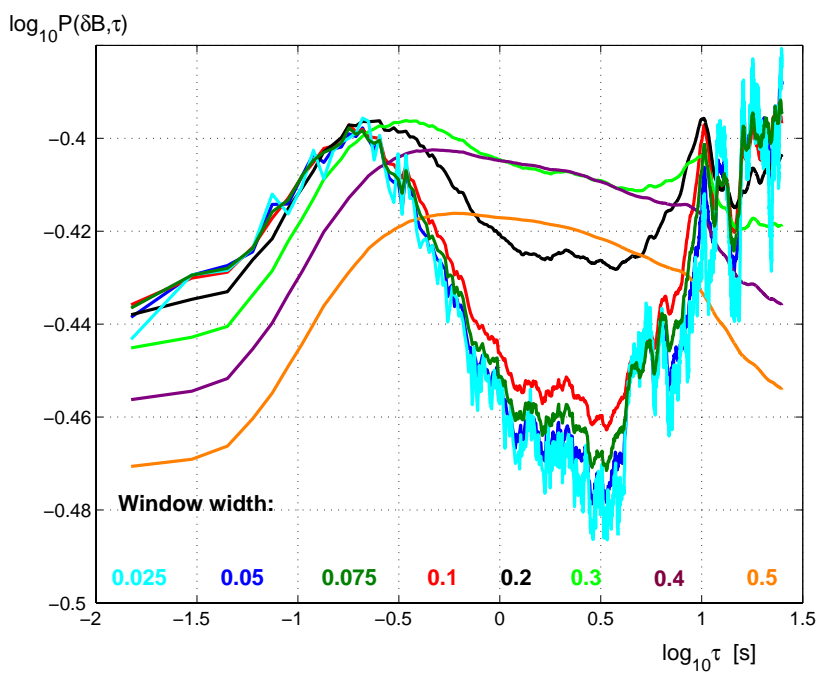

Fig. 3. Dependence of $P(\delta B, \tau)$ on window width (Eq. 1$) ; h=0.05$ is chosen for further analysis.

patterns in the double-logarithmic plot of $P(\delta B, \tau)$ versus $\tau$ which can be regarded as specific signatures of small-scale turbulent fluctuations (see later). Therefore, our interest is not in PDF rescaling techniques, but in identifying multiscale patterns of $P(\delta B, \tau)$.

Figure 3 shows the double logarithmic plots of $P(\delta B, \tau)$ versus $\tau$ for the magnitude of the magnetic field ( $B_{X}$ is depicted in Fig. 1a). Here and hereinafter in similar plots we consider time scales $\tau$ from $1 / 67 \mathrm{~s}$ to $25 \mathrm{~s}$ with a step of $1 / 67 \mathrm{~s}$. The multi-scale evolution of a point next to the maxima of PDFs is followed. The number of data points is fixed to 200000 but the window width $h$ is changed from 0.025 to 0.5 . When $h$ is chosen too small ( $h=0.025$ in our case) spurious fine structures and fluctuations appear. If $h$ is too large $(h \geq 0.2)$ then the fine structure between the scales $\log _{10} \tau \in(-0.5,1.5)$ is entirely obscured. It is very important to notice that for $h \leq 0.1$ all the curves obtained from magnetic PDFs are nearly coincident in Fig. 3. For further analysis $h=0.05$ is chosen.

Figure 4 shows the dependence of $P(\delta B, \tau)$ on the number of data points $n$. Always the first $n=12500,25000,50000, \ldots, 200000$ points are used from the magnetic data, whilst $h=0.05$ is fixed. The curves exhibit large fluctuations particularly for the shortest intervals with $n=12500,25000$ at the largest time scales $\log _{10} \tau \geq 0$. Besides that, different patterns appear in the double logarithmic plots of $P(\delta B, \tau)$ versus $\tau$ because of the large velocity variations (from 50 and $1000 \mathrm{~km} / \mathrm{s}$ in Fig. 1b) during the analysed intervals. It is also the sign of the expected velocity dependence of PDFs shapes, mentioned above. Consequently, the robustness of the estimations against $n$ should be tested for intervals with small changes in large-scale bulk velocity. As it was mentioned, however, high-speed flows last not longer than a few minutes. Nonetheless, one can find longer steady intervals of low-speed flows. Figure 5 shows the dependence of $P(\delta B, \tau)$ on the number of data points $n$

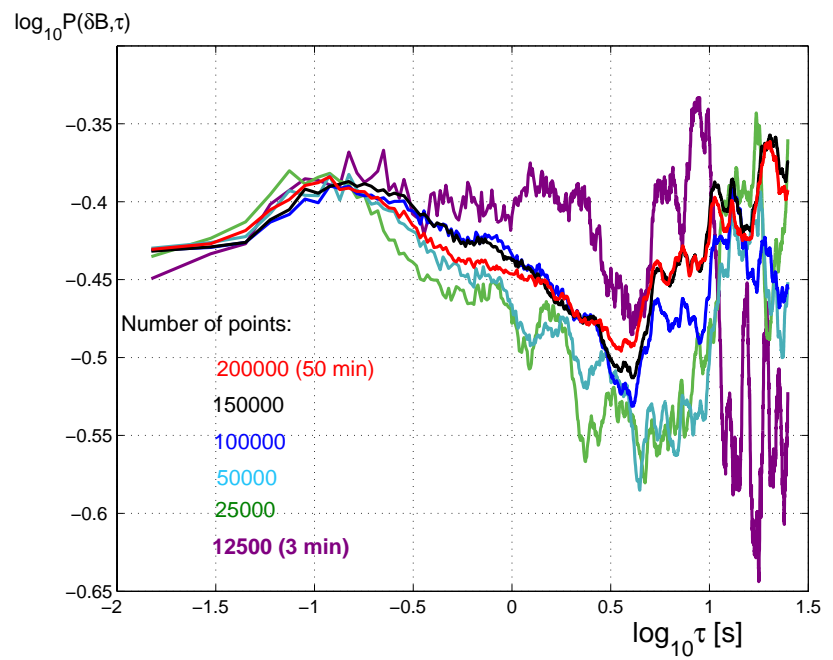

Fig. 4. Dependence of $P(\delta B, \tau)$ on the number of data points; $n=25000(6 \mathrm{~min})$ is chosen for further analysis.

for a $25 \mathrm{~min}$ long steady interval on 7 September 2001, from 18:18 to 18:43 UT (not shown), s/c 3, when the plasma velocity was $V=42 \pm 8 \mathrm{~km} / \mathrm{s}$. The number of data points does not influence the shape of the curves. Only the largest time scales ( $>10 \mathrm{~s}$ ) show different behaviour for the longest interval (100 000 points $\sim 25 \mathrm{~min}$ ) which is a consequence of nonstationarity of the magnetic field (not shown). The largest fluctuations occur for the shortest interval of 12500 points $(\sim 3 \mathrm{~min})$. Therefore the choice to analyse $6 \mathrm{~min}$ long intervals of magnetic data seems to be a reasonable trade-off between the precision and the required steadiness of flows.

It is easy to interpret the emerging patterns visible in Fig. 5. The peaks at the time scales larger than $1 \mathrm{~s}$, up to $24 \mathrm{~s}$, are harmonics appearing due to the modulation of the time series $\delta B$ (Eq. 1) by the spin-tone ( $4 \mathrm{~s})$. The scaling region with the scaling index $\alpha \sim 0.17$ in Eq. (3) is the property of magnetometer noise, profoundly analysed in Vörös et al. (2004b). The breaking of that scaling at about $0.1 \mathrm{~s}$ comes from the finite accuracy and resolution of magnetic measurements influenced also by the window width $h$.

Though the first $2 \mathrm{~s}$ peak and its multiples can be occassionally suppressed because of the changing angle between the magnetic field and the spacecraft spin axis, whenever the plasma flow velocity in the plasma sheet is below a threshold (a few tens of $\mathrm{km} / \mathrm{s}$ ) the basic pattern presented in Fig. 5 is repeatedly observed. It indicates that in such cases, at least over the range of time scales from $2 / 67 \mathrm{~s}$ to $25 \mathrm{~s}$, cascades or any other types of multi-scale coupling are not present. Therefore, in what follows, we will refer to the basic pattern in Fig. 5 as to a "non-flow" pattern.

\section{More patterns}

Our goal is to investigate how the presence of a rapid flow and a turbulent cascade can influence the basic non-flow pat- 


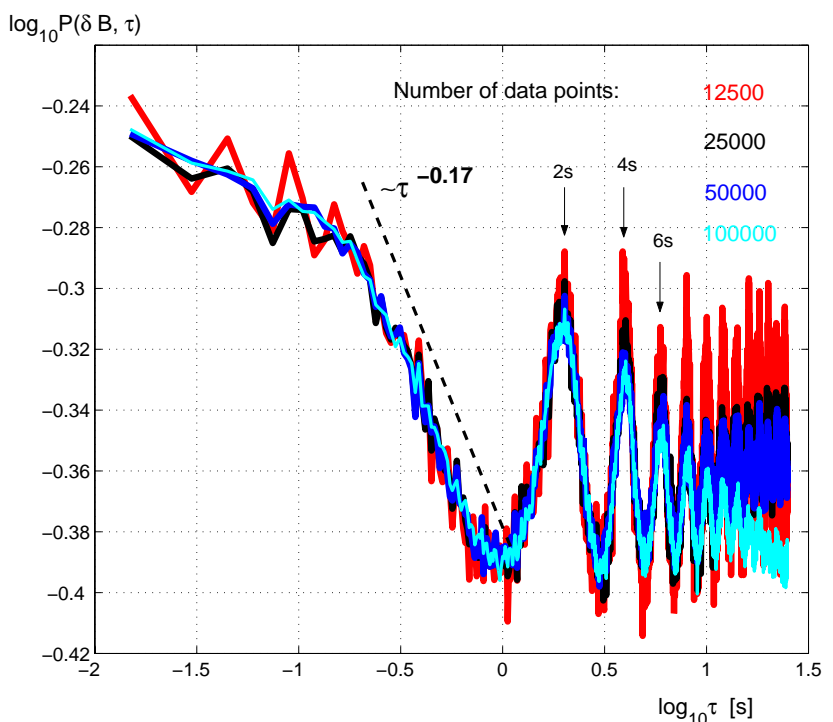

Fig. 5. Robustness of the basic "non-flow" pattern against $n$.

tern of $P(\delta B, \tau)$ in Fig. 5. A comparison between Figs. 4 and 5 already reveals that rapid flows obscure spin-tone harmonics or noise related scaling. Certainly, the flow energy cascades towards large wavenumbers and when the dissipation scale is reached, the energy is converted into heat. The shapes of the two-point PDFs (Eqs. 1 and 3) estimated from the magnetic field will be definitely influenced by a cascade within the inertial range or within the corresponding time scales $\tau$. On the contrary, there is no reason to expect that the magnetic fluctuations below the dissipation scale or the corresponding PDFs would be strongly affected by the inertial range cascade. In other words, if $\tau_{d}$ denotes the dissipation time scale, for the scales $\tau>\tau_{d}$ the basic non-flow pattern in Fig. 5 will be obliterated, while the scales $\tau<\tau_{d}$ remain unchanged.

From now on we select 6 min long intervals for further study. It is approximately the time-scale of the typically observed groups of high-speed intermittent flows. We select quasi-stationary intervals whilst the mean bulk velocity computed from sequential spin resolution data is reasonably stable. Each interval contain 24120 data points from magnetic field and 90 data points from plasma velocity measurements.

Figure 6 shows such a case where $\tau_{d} \sim 5 \mathrm{~s}$ and over the scales $\tau>5 \mathrm{~s}$ all the spin-tone harmonics disappear, while for the scales $\tau<5 \mathrm{~s}$ the pattern is the same as in Fig. 5. The relationship $P(\delta B, \tau)$ versus $\tau$ in Fig. 6 was estimated for the 6 min long interval from 19:15 to 19:21 UT on 7 September 2001 , s/c 3, when the plasma velocity was $V=70 \pm 20 \mathrm{~km} / \mathrm{s}$. The four curves in Fig. 6 reflect the scale dependence of four successive points on the positive part of the PDF curve $(\delta B>0)$ next to its maximum. Skewness is neglected, PDFs are supposed to be symmetric. The density is estimated at 80 equally-spaced points covering the range of the data set. The maxima of the PDFs were always at or near the center of the data range.

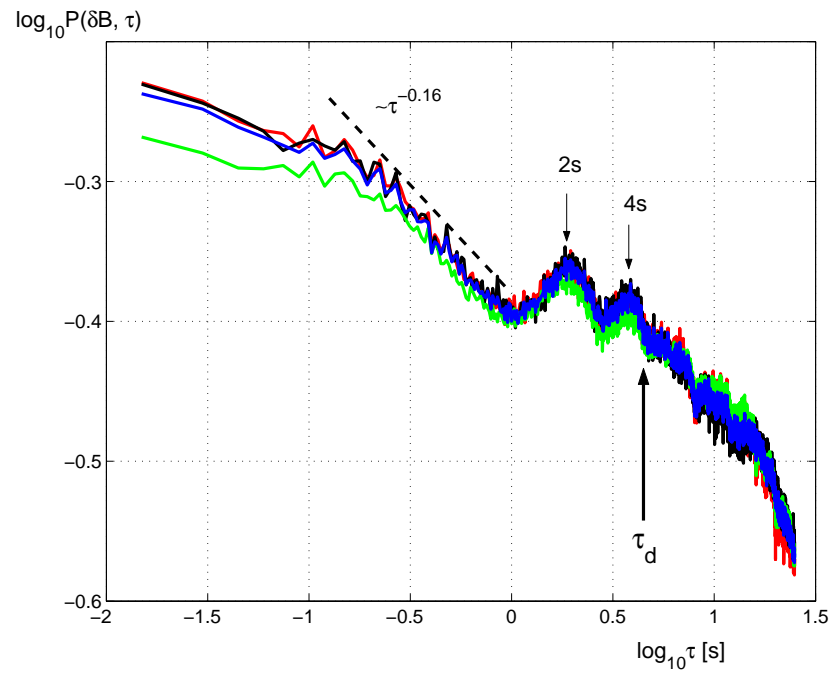

Fig. 6. Slow-flow associated pattern; $\tau_{d}$ denotes the estimated dissipation scale.

A simple method of finding $\tau_{d}$ is to compute the crosscorrelation between the four curves and the so-called pvalues for testing the hypothesis of no correlation. Near the $2 \mathrm{~s}$ and $4 \mathrm{~s}$ peaks each pair of the curves (altogether 6 pairs) is highly correlated $(p<0.05)$ whereas at the scales $\tau>\tau_{d}$ the correlation is lost $(p \gg 0.05)$. From the 6 pairs of crosscorrelated curves we found $\tau_{d}=5 \pm 1 \mathrm{~s}$. This method for finding the dissipation time-scale can be used when $\tau_{d} \geq 2 \mathrm{~s}$ and the disappearance of the spin-tone harmonics indicates the presence of low-speed flow associated multi-scale fluctuations.

In case of high-speed flows usually $\tau_{d}<2 \mathrm{~s}$ is found (see later) and the emerging patterns in $P(\delta B, \tau)$ are the result of the interaction of bulk flow associated multi-scale fluctuations and magnetometer noise (basic non-flow pattern in Fig. 5). Within this range of time scales spin-tone harmonics are not present. Yet, using a similar model as in Vörös et al. (2004b) the dissipation time-scale can be found also in this case. We generate a model consisting of an arbitrary artificial multi-scale signal (AMS) added to the magnetometer noise. AMS mimics certain properties of multi-scale turbulence. It is important to note that we are not interested in the exact scaling properties of AMS. We simply ask how the basic non-flow pattern in Fig. 5 changes when all the fluctuations in AMS, added to the magnetometer noise, are smoothed out below a time scale $\tau_{d}^{*}$. In other words, at scales $\tau>\tau_{d}^{*}$ multiscale fluctuations are present in AMS resembling the inertial range fluctuations. The "cascade" is artificially stopped at $\tau_{d}^{*}$ and there are only smooth variations at the scales $\tau<\tau_{d}^{*}$. As for AMS, a multifractal signal can be used, which is constructed on the basis of a recursive rule and is also well suited for modeling intermittency in the plasma sheet (Vörös et al., 2003). Another possibility for AMS is to generate a synthetic fractal signal, e.g. a fractional Brownian motion (fBm) through a fast filter bank based pyramidal algorithm (Abry 


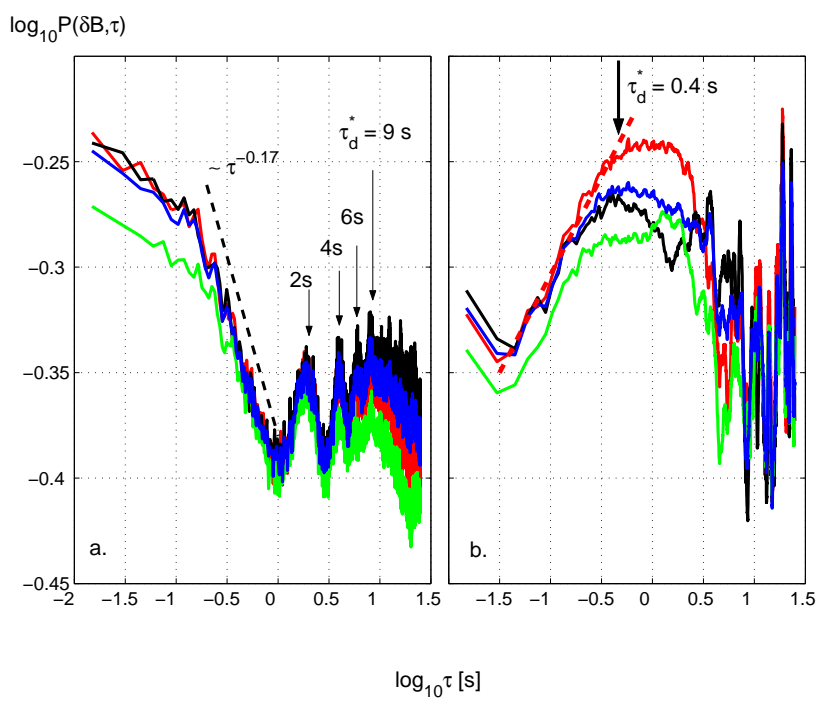

Fig. 7. Model patterns for (a) slow flows, (b) rapid flows; $\tau_{d}^{*}$ denotes the artificial dissipation scale, where a "cascade" is stopped.

and Sellan, 1996). In both cases $\tau_{d}^{*}$ can be changed arbitrarily resulting in similar patterns which then can be compared to experimental patterns obtained from magnetic data. Since AMS can model turbulence only approximately, we only aim at understanding the qualitative changes in $P(\delta B, \tau)$ when the model $\tau_{d}^{*}$ is altered.

Figure $7 \mathrm{a}$ shows the case when a model $\mathrm{fBm}$ was added to the steady low-speed associated interval on 7 September 2001. The model $\tau_{d}^{*}$ was $9 \mathrm{~s}$ and $P(\delta B, \tau)$ resembles the patterns in Fig. 6. Again, over the scales larger than $\tau_{d}^{*}$ all the spin-tone harmonics cease, while for the scales smaller than $\tau_{d}^{*}$ the patterns are the same as in the case of noise in Fig. 5.

Figure $7 \mathrm{~b}$ demonstrates the alteration of the model pattern for $\tau_{d}^{*}=0.4 \mathrm{~s}$. The basic non-flow pattern from Fig. 5, consisting of the noise scaling range and the spin-tone harmonics range is entirely absent. The scales larger than a few seconds (right hand part of Fig. 7b) contain large fluctuations because of pure statistics. At the scales smaller than the model dissipation time-scale $\tau_{d}^{*}$, all the curves show rapid power-law decrease. When the model $\tau_{d}^{*}$ increases (the cascade is stopped at a larger time scale) the rapidly decreasing power-law region widens towards large scales and vice versa (not shown).

On the basis of the model examples we expect that whenever the bulk velocity (or the Reynolds number) of the flow increases, the inertial range widens, the dissipation range reaches smaller time scales and its approximate value, $\tau_{d}$, can be estimated from the emerging patterns of $P(\delta B, \tau)$.

The resemblance between a low-speed associated pattern (Fig. 6) and the corresponding model pattern with a dissipation time scale within the range of spin-tone harmonics (Fig. 7a) has already been shown. Let us demonstrate now the similarity between a high-speed flow pattern (Fig. 8) and the model pattern with artificial fluctuations stopped at the scale $\tau_{d}^{*}=0.4 \mathrm{~s}$ (Fig. 7b). The curves in Fig. 8 correspond

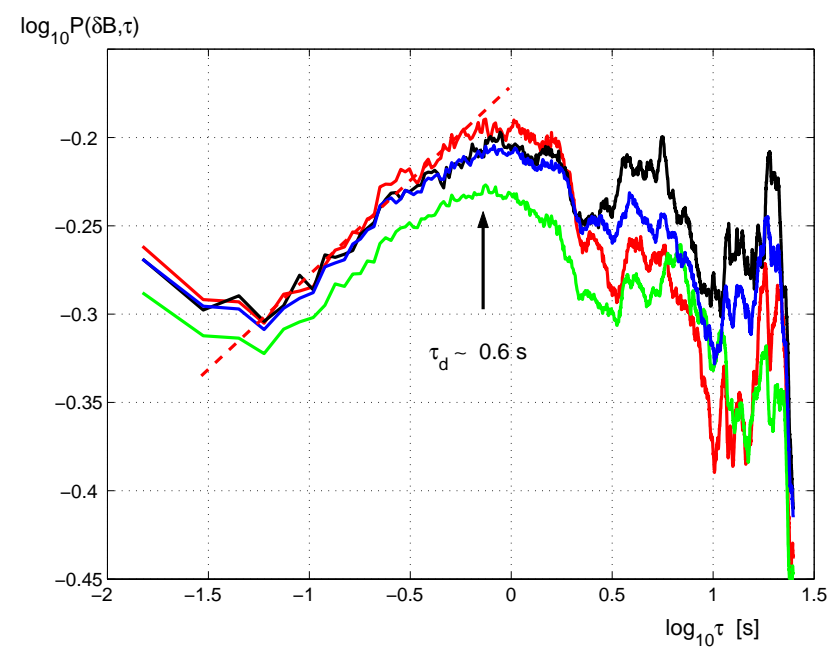

Fig. 8. Rapid-flow associated pattern; $\tau_{d}$ denotes the estimated dissipation scale.

to the interval on 27 August 2001, from 01:35 to 01:41 UT when the bulk velocity was $V=550 \pm 170 \mathrm{~km} / \mathrm{s}$ at $\mathrm{s} / \mathrm{c} 3$. The patterns in $P(\delta B, \tau)$ obtained from magnetic data exhibit similar shapes as in the model case, large fluctuations at the largest scales and a sudden power-law decrease at the small scales. The dissipation time-scale can be estimated at the small scales when the curves suddenly start decreasing, following a power-law. It is a feature typically observed in cases of high-speed flow associated PDFs. $\tau_{d}$ can be find as an instant when large departures are observed from the power-law fit. On this basis we found $\tau_{d}=0.7 \pm 0.15 \mathrm{~s}$. The errors are computed from the four curves only, yet they provide helpful insights about the stability of the PDF shape near its maximum.

\section{Multi-scale coupling}

In the previous section we identified multi-scale patterns of alterations of PDFs shapes corresponding to magnetic fluctuations during both low-speed and high-speed flows. In order to explore the bulk velocity dependence of the disspiation time-scale we select 6 min long intervals in the plasma sheet, during which the velocity fluctuations are much smaller than their mean value. We considered 3 longer intervals on 27 August 2001, between 01:35 and 02:25 UT, on 7 September 2001, between 19:00 and 20:30 UT and on 15 September 2001, between 03:30 and 04:10 UT, which contain several steady flow subintervals. Using burst mode magnetic and spin resolution velocity data from the s/c 1, 3, 4 spacecraft (there were no velocity measurements in $\mathrm{s} / \mathrm{c} 2$ ) we found 50 , 6 min long subintervals which satisfied the selection criteria. 9 intervals were excluded from the data analysis, because of the lack of magnetic fluctuations over the examined range of scales or ambiguities in determination of the dissipation time-scale. For the remaining 41 events the mean value and the standard deviation were computed for the bulk veloc- 
ity $V$ and the dissipation magnetic time-scale $\tau_{d}$. The large plot in Fig. 9 shows the result which can be approximated, within the range of values $V \in(30,800) \mathrm{km} / \mathrm{s}$, with powerlaw $\tau_{d}=0.4+150000 V^{-2.6}$. Rapid flows exhibit larger velocity fluctuations than slow flows, but the slow-flow associated events have larger uncertainities in $\tau_{d}$. The latter can be the result of an approximate mapping of spatial structures through Equation 2 during slow flows. We note that $\tau_{d}$ changes substantially between $V=50$ and $200 \mathrm{~km} / \mathrm{s}$, then $\tau_{d}$ reaches asymptotically $\sim 0.4 \mathrm{~s}$. The smallest value in the analysed data set was $\tau_{d}=0.1 \pm 0.05 \mathrm{~s}$. The overlaying inset in Fig. 9 shows the range of $\tau_{d} \in(0,2)$ enlarged.

\section{Conclusions}

Hydrodynamic or magnetohydrodynamic turbulent flows are rich of coherent structures and self-similar fluctuations arising in consequence of the redistribution of flow energy introduced typically at large (system) scales.

In this paper, for the first time, we found a quantitative relationship between the large-scale mean flow (quasi-steady bulk velocity during 6 min intervals) and the dissipation scale in the plasma sheet. The dissipation scale was estimated on the basis of a new technique which classifies the emerging patterns of PDFs near their maxima at different time scales. It was found that the dissipation scale is shifted towards smaller scales when the bulk velocity (flow Reynolds number) increases. This is a generic behaviour observed in many turbulent flows, which also substantiates previous conjectures that the observed multi-scale fluctuations in the plasma sheet, over the range from seconds to minutes, arise due to the strongly dissipative turbulence rather than waves or uncorrelated noise. Obviously, it does not exclude the possibility of simultaneous occurrence of turbulence, waves or noise over the same scale ranges in the plasma sheet.

It has already been noticed by Angelopoulos et al. (1999) that the magnetotail plasma is in a bi-modal state: highly intermittent during transport-efficient fast flows and nearly stagnant otherwise. We found that, during the $6 \mathrm{~min}$ long intervals, magnetic fluctuations and the associated patterns of PDFs, are velocity dependent and exceedingly different for high-speed and low-speed flows. During longer time intervals fast flows are embedded into low-speed or stagnant intervals. It also means that intermittency has a different meaning at different spatial and temporal scales. At the time scales from minutes down to a fraction of a second (dissipation time-scale) intermittency is related to the turbulence inside a flow channel (e.g. Vörös et al., 2003). The largest time-scale is determined as the actual time of merging of the spacecraft into a channel, containing a turbulent flow. On the other side, time scales from minutes to hours can include several BBFs, separated by stagnant low-speed inter-flow intervals. Intermittency observed over these spatial and temporal scales can be related mainly to the impulsive driving mechanisms of BBFs, e.g. to the distant transient (Sergeev et al., 1987) or multiple (Slavin et al., 2003) reconnection-sites. Therefore

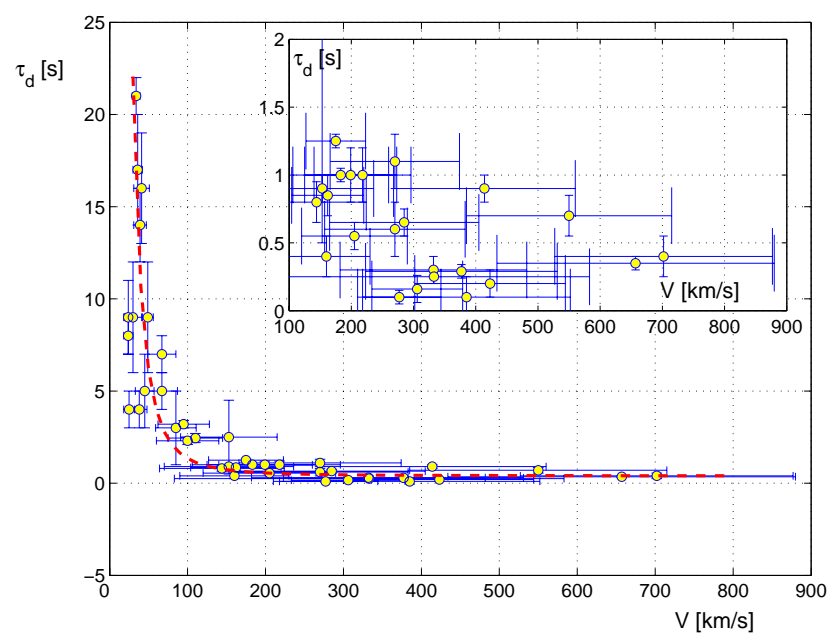

Fig. 9. Main plot: dissipation time-scale versus bulk velocity estimated for 41 intervals using Cluster s/c 1, 3, 4 data from 2001; Inset: enlargement of the dissipation scale range $\tau_{d} \in(0,2) \mathrm{s}$.

any analysis or modeling of turbulence should necessarily reflect the scale-dependent diversity of physical processes in the plasma sheet.

Acknowledgements. We thank Y. Asano, T. Takada and T. Zhang for helpful discussions and comments. The authors are grateful to H. U. Eichelberger for helping in the Cluster data analysis.

Edited by: D. Vassiliadis

Reviewed by: R. Treumann and two referees

\section{References}

Abry, P. and Sellan, F.: Some remarks on and fast implementation for the wavelet-based synthesis of fractional Brownian motion proposed by Sellan, F. and Meyer, Y., Appl. Comput. Harm. Anal., 3, 377-383, 1996.

Angelopoulos, V., Baumjohann, W., Kennel, C., Coroniti, F., Kivelson, M., Pellat, R., Walker, R., and Lühr, H.: Bursty bulk flows in the inner central plasma sheet, J. Geophys. Res., 97, 4027-4039, 1992.

Angelopoulos, V., Mukai, T., and Kokubun, S.: Evidence for intermittency in Earth's plasma sheet and implications for selforganized criticality, Phys. Plasmas, 6, 4161-4168, 1999.

Balogh, A., Carr, C., Acuña, M., Dunlop, M., Beek, T., Brown, P., Fornacon, K.-H., Georgescu, E., Glassmeier, K.-H., Harris, J., Musmann, G., Oddy, T., and Schwingenschuh, K.: The Cluster magnetic field investigation: Overview of inflight perfomance and initial results, Ann. Geophys., 19, 1207-1217, 2001.

SRef-ID: 1432-0576/ag/2001-19-1207.

Baumjohann, W., Paschmann, G., and Lühr, H.: Characteristics of high-speed ion flows in the plasma sheet, J. Geophys. Res., 95, 3801-3810, 1990.

Bershadskii, A. and Sreenivasan, K.: Intermittency and the passive nature of the magnitude of the magnetic field, Phys. Rev. Lett., 93, 064 501-1-064 501-4, 2004. 
Borovsky, J. and Funsten, H.: MHD turbulence in the Earth's plasma sheet: Dynamics, dissipation and driving, J. Geophys. Res., 108, 1284, doi:10.1029/2002JA009 625, 2003.

Borovsky, J., Elphic, R., Funsten, H., and Thomsen, M.: The Earth's plasma sheet as a laboratory for flow turbulence in highbeta MHD, J. Plasma Phys., 57, 1-34, 1997.

Bruno, R., Carbone, V., Primavera, L., Sorriso-Valvo, L., Bavassano, B., and Veltri, P.: On the probability distribution functions of small-scale interplanetary magnetic field fluctuations, Ann. Geophys., 22, 3751-3769, 2004.

\section{SRef-ID: 1432-0576/ag/2004-22-3751}

Chang, T.: Self-organized criticality, multi-fractal spectra, sporadic localized reconnections and intermittent turbulence in the magnetotail, Phys. Plasmas, 6, 4137-4145, 1999.

Consolini, G. and Chang, T.: Magnetic field topology and criticality in Geotail dynamics: Relevance to substrom phenomena, Space. Sci. Rev., 95, 309-321, 2001.

Frisch, U.: Turbulence, Cambridge University Press, Cambridge, 1995.

Greco, A., Veltri, P., Zimbardo, G., Taktakishvili, A., and Zelenyi, L.: Numerical simulation of ion dynamics in the magnetotail magnetic trubulence: on collisionless conductivity, Nonlin. Processes Geophys., 7, 159-166, 2000.

SRef-ID: 1607-7946/npg/2000-7-159.

Hnat, B., Chapman, S., Rowlands, G., Watkins, N., and Farrell, W.: Finite size scaling in the solar wind magnetic field energy density as seen by Wind, Geophys. Res. Lett., 29, 1446, doi:10.1029/2001GL014 587, 2002.

Hnat, B., Chapman, S., and Rowlands, G.: Intermittency, scaling and the Fokker-Planck approach to fluctuations of the solar wind bulk plasma parameters as seen by the Wind spacecraft, Phys. Rev. E, 676, 056 404, doi:10.1103/PhysRevE.67.056 404, 2003.

Horbury, T. and Balogh, A.: Evolution of magnetic field fluctuations in high-speed solar wind streams: Ulysses and Helios observations, J. Geophys. Res., 106, 15 929-15 940, 2001.

Klimas, A., Uritsky, V., Vassiliadis, D., and Baker, D.: Reconnection and scale-free avalanching in a driven current sheet model, J. Geophys. Res., 109, doi:10.1029/2003JA010 036, 2004.

Leubner, M. and Vörös, Z.: A nonextensive entropy approach to solar wind intermittency, Astrophys. J., 618, 547-555, 2005a.

Leubner, M. and Vörös, Z.: A nonextensive entropy path to probability distributions in solar wind turbulence, Nonlin. Processes Geophys., 12, 171-180, 2005b,

\section{SRef-ID: 1607-7946/npg/2005-12-171.}

Nakamura, R., Baumjohann, W., Mouikis, C., Kistler, L., Runov, A., Volwerk, M., Asano, Y., Vörös, Z., Zhang, T., Klecker, B., Rème, H., and Balogh, A.: Spatial scale of high-speed flows in the plasma sheet observed by Cluster, Geophys. Res. Lett., 31, L09 894, doi:10.1029/2004GL019558, 2004.

Neagu, E., Borovsky, J., Thomsen, M., Gary, S., Baumjohann, W., and Treumann, R.: Statistical survey of magnetic field and ion velocity fluctuations in the near-Earth plasma sheet: Active Magnetospheric Particle Trace Explorers/Ion Release Module (AMPTE/IRM) measurements, J. Geophys. Res., 107, doi:10.1029/2001JA000 318, 2002.

Oughton, S.: Solar wind fluctuations: Waves and turbulence, in Proceedings of the Tenth International Solar Wind Conference, edited by M. Velli, R. Bruno, and F. Malara, 421-426, American Institute of Physics, Maryland, 2003.

Porta, A. L., Voth, G., Crawford, A., Alexander, J., and Bodenschatz, E.: Fluid particle accelerations in fully developed turbulence, Nature, 409, 1017-1019, 2001.
Rème, H., Aostin, C., Bosqued, J., Danduras, I., Lavraud, B., Sauvaud, J., Barthe, A., Bouyssou, J., Camus, T., Coeur-Joly, O., et al.: First multispacecraft ion measurements in and near the Earth's magnetosphere with the identical Cluster ion spectrometry (CIS) experiment, Ann. Geophys., 19, 1303-1354, 2001, SRef-ID: 1432-0576/ag/2001-19-1303.

Saddoughi, S. and Veeravalli, S.: Local isotropy in turbulent boundary layers at high Reynolds number, J. Fluid. Mech., 268, 333372, 1994.

Schödel, R., Baumjohann, W., Nakamura, R., Sergeev, V., and Mukai, T.: Rapid flux transport in the central plasma sheet, J. Geophys. Res., 106, 301-313, 2001.

Sergeev, V., Semenov, V., and Sidneva, M.: Impulsive reconnection in the magnetotail during substorm expansion, Planet. Space Sci., 35, 1199-1212, 1987.

Silverman, B.: Density estimation for statistics and data analysis, Chapman and Hall, London, 1986.

Slavin, J., Owen, C. J., Dunlop, M. W., Borälv, E., Moldwin, M. B., Sibeck, D. G., Tanskanen, E., Goldstein, M. L., Fazaderley, A., Balogh, A., Lucek, E., Richter, I., Rème, H., and Bosqued, J.: Cluster four spacecraft measurements of small traveling compression regions in the near-tail, Geophys. Res. Lett., 30, 2208 , doi:10.1029/2003GL018 438, 2003.

Sornette, D.: Critical Phenomena in natural sciences, SpringerVerlag, Berlin, 2000.

Sorriso-Valvo, L., Carbone, V., and Veltri, P.: Intermittency in the solar wind turbulence through probability distribution functions, Geophys. Res. Lett., 26, 1801-1804, 1999.

Tu, C. and Marsch, E.: MHD structures, waves and turbulence in the solar wind: Observations and theories, Space. Sci. Rev., 73, 1-210, 1995.

Volwerk, M., Nakamura, R., Baumjohann, W., Treumann, R., Runov, A., Vörös, Z., Zhang, T., Asano, Y., Klecker, B., Richter, I., Balogh, A., and Rème, H.: A statistical study of compressional waves in the tail current sheet, J. Geophys. Res., 108, 1429, doi:10.1029/2003JA010 155, 2003.

Volwerk, M., Vörös, Z., Baumjohann, W., Nakamura, R., Runov, A., Zhang, T., Glassmeier, K.-H., Treumann, R., Klecker, B., Balogh, A., and Rème, H.: Multi-scale analysis of turbulence in the Earth's current sheet, Ann. Geophys., 22, 2525-2533, 2004. SRef-ID: 1432-0576/ag/2004-22-2525

Vörös, Z., Baumjohann, W., Nakamura, R., Runov, A., Zhang, T., Volwerk, M., Eichelberger, H.-U., Balogh, A., Horbury, T., Glassmeier, K.-H., Klecker, B., and Rème, H.: Multi-scale magnetic field intermittence in the plasma sheet, Ann. Geophys., 21, 1955-1964, 2003. SRef-ID: 1432-0576/ag/2003-21-1955

Vörös, Z., Baumjohann, W., Nakamura, R., Runov, A., Volwerk, M., Zhang, T., and Balogh, A.: Wavelet analysis of magnetic turbulence in the plasma sheet, Phys. Plasmas, 11, doi:10.1063/1.1667 499, 2004a.

Vörös, Z., Baumjohann, W., Nakamura, R., Volwerk, M., Runov, A., Zhang, T., Eichelberger, H.-U., Treumann, R., Georgescu, E., Balogh, A., Klecker, B., and Rème, H.: Magnetic turbulence in the plasma sheet, J. Geophys. Res., 109, A11215, doi:10.1029/2004JA010 404, 2004b.

Weygand, J., Kivelson, M., Khurana, K., Schwarzl, H., Thompson, S., McPherron, R., Balogh, A., Kistler, L., Goldstein, M., Borovsky, J., and Roberts, D.: Plasma sheet turbulence observed by Cluster II, J. Geophys. Res., 110, A01 205, doi:10.1029/2004JA010 581, 2005 